\title{
Docencia de la investigación en la universidad actual
}

Teaching of research at the current university

Dr. Luis Rodríguez de los

Ríos

Universidad Nacional de

Educación del Perú

Recepción

2/ 03/2018

Revisado

9/04/2018

Aceptación

26/08/2018

\section{Resumen}

7 xiste un cambio de paradigmas en la Educación Superior que implica un paso de la sociedad de información a la sociedad de conocimiento, lo cual deriva en un cambio en el rendimiento educativo a nivel global. Se prioriza la investigación básica y tecnológica y la colaboración interinstitucional e interdisciplinar. La Ley Universitaria $\mathrm{N}^{\circ} 30220$ le asigna una importancia decisiva desde que estipula un Vicerrectorado de Investigación. Una de las varias maneras de impulsar la investigación, así ubicada, es mediante políticas específicas de implementación y desarrollo de docencia para la investigación, desde el pregrado hasta el posdoctoral. Se impone la investigación para el desarrollo y la innovación y la colaboración con las empresas para la formación de competencias laborales y el emprendimiento.

Palabras clave: sociedad del conocimiento, investigación básica y tecnológica, docencia, investigación, interdisciplinaria.

\section{Abstract}

7 here is a paradigm shift in higher education with the shift from the information society to the knowledge society and the measurement of its performance globally. Priority is given to basic and technological research and inter-institutional and interdisciplinary collaboration. The University Law $\mathrm{N}^{\circ} 30220$ assigns a decisive importance to him since stipulates a vice-rector of investigation. One 
of several ways to promote the research so located is through specific policies of implementation of teaching development for research from undergraduate to postdoctoral. Research is required for development and innovation and collaboration with companies for the training of job skills and entrepreneurship.

Key words: knowledge society, basic and technological research, teaching, research, interdisciplinary.

\section{Introducción}

Hoy en día, cuando se habla del proceso docente educativo en las universidades, este debe ajustarse plenamente a los cambios y transformaciones que van corriendo aceleradamente en la sociedad. Por tanto, el concepto amplio de universidad no se circunscribe solamente a la docencia pura ejercida en las aulas. El diapasón cultural se amplía, entre otras cosas a la relación sujetosujeto donde el alumno no debe convertirse en un ser pasivo subordinado a las orientaciones del profesor; entonces debe cambiar su papel y convertirse también en sujeto del conocimiento, es decir, formar parte del protagonismo de ese proceso, claro está, con la guía orientadora del profesor.

De lo anterior se deriva que cuando nos referimos al papel de las universidades, se debe hacer más énfasis en el aprendizaje que en la enseñanza, en un proceso dialógico donde también se tengan en cuenta otros componentes como el investigativo.

Este criterio se ha generalizado en los pedagogos de vanguardia de los cuales ofrecemos algunos ejemplos.

Desde hace más de dos décadas, los sistemas de educación superior de todo el mundo vienen experimentando un conjunto de transformaciones, signadas por la apuesta en favor de la calidad, la pertinencia, la inclusión, la equidad y la internacionalización. En un informe de (UNESCO-ESALC,2018), en función de las megatendencias socioeconómicas postmodernas y de las nuevas tendencias pedagógicas; enmarcadas en cambios de gran calado denominado como: sociedad del conocimiento, es decir con capacidad para 
identificar, producir, tratar, transformar, difundir y utilizar la información con vistas a crear y aplicar los conocimientos necesarios para el desarrollo humano (Unesco, 2005, p. 29.).

La Sociedad del Conocimiento alude a la presencia de un nuevo paradigma económico - productivo en el cual el factor más importante no es ya la disponibilidad de capital, mano de obra, materias primas o energía, sino el uso intensivo del conocimiento y la información (Tünnermann y de Sousa Chaui, 2003).

El desarrollo de la economía y las organizaciones en general, actualmente, dependen de la accesibilidad y disponibilidad del conocimiento, del manejo competitivo del mismo y de las innovaciones tecnológicas. De allí, la importancia que ha asumido la generación de nuevos conocimientos a través de procesos de investigación, en general, $y$, de la investigación científica en particular, en el entendido de que la producción de conocimientos nuevos es el motor fundamental para el aumento de la riqueza y el bienestar social (Quintanilla, 2007).

En este sentido, el conocimiento como característica de las sociedades actuales tiene implicancias sobre la educación y sobre las políticas de investigación y desarrollo, constituyéndose en todo un desafío para la educación superior, en especial para la educación universitaria. Si bien una de las funciones de la universidad, es la investigación, nunca antes tuvo la prioridad y trascendencia como en los últimos tiempos. Ello lo podemos evidenciar en todas las leyes de reforma de los sistemas de educación que se vienen implementando a nivel mundial en las cuales se especifica claramente como misión fundamental de la universidad la de generar, aplicar, transferir y divulgar los conocimientos nuevos.

Recientemente, la producción científica de las universidades es medida por la cantidad de publicaciones arbitradas y acreditadas, las cuales constituyen un criterio importante para la inclusión en los rankings a nivel nacional, regional y mundial.

De igual manera, la investigación en las universidades tanto de América del Norte, Europa, Asia y Latinoamérica son referentes para tomar conciencia de la disparidad y calidad existentes en el nivel de desarrollo. 
Es por ello que para otorgar una categoría, coexisten universidades donde se desarrollan investigaciones de alta calidad, de mediana y baja calidad.

Las diferencias se ubican en cuanto a políticas institucionales, infraestructura, equipos, financiamiento, publicaciones indizadas, existencia de comunidades científicas y existencia de una cultura de investigación fortalecida y sostenida; asociada, predominantemente, a la docencia investigativa y a hacer la investigación vinculada al objetivo supremo de la formación de un egresado más capaz.

\section{Contextualización.}

En el año 2006, el Ministerio de Educación publicó el informe: La universidad en el Perú. Razones para una Reforma Universitaria; en el cual se hacía un diagnóstico de la realidad universitaria en todas sus dimensiones. La investigación sostenía lo siguiente:

La producción de conocimiento, función esencial de la universidad, se halla muy relegada, particularmente en el área estratégica de las ciencias básicas y la producción de la tecnología. No hay lineamientos generales, de alcance nacional, que coordinen la investigación en las universidades, fijando prioridades $\mathrm{y}$ división de funciones al menos entre las universidades públicas, situación que suele repetirse en cada universidad. La ausencia de políticas coherentes de investigación de largo plazo e impacto social, que favorezcan la interdisciplinariedad y la cooperación entre universidades, impide la conformación de una comunidad científica, académica e intelectual integrada a redes de cooperación permanentes. Ello da cuenta del aislamiento de la universidad con respecto a su entorno, en particular con el sector productivo y el Estado; pero es ante todo resultado de la escasa atención puesta por los gobiernos de turno al desarrollo de nuestra base científico tecnológica. (p. 84).

En el mes de julio del año 2014, se promulgó la Ley 30220, la misma que en su Artículo 1 define su objeto: La presente Ley tiene por objeto normar la creación, funcionamiento, supervisión y cierre de las universidades. Promueve el mejoramiento continuo de la calidad educativa de las instituciones 
universitarias como entes fundamentales del desarrollo nacional, de la investigación y de la cultura.

Su pretensión principal es fortalecer y potenciar la labor investigativa en las universidades, con el propósito de remontar las debilidades señaladas en el párrafo anterior, dedicándole cerca de catorce artículos para su fomento y desarrollo: Artículos 6, 7, 11, 15, 30, 31, 37, 48, 49, 50, 65, 86, 87, 88.

Específicamente el Art. 65, está dedicado al Vicerrectorado de investigación como órgano de la universidad creado a partir de la Ley, reemplazando al Vicerrectorado Administrativo establecido en la Ley anterior. Al respecto, consideramos que lo normado en la nueva Ley sobre el proceso de investigación es un marco de actuación importante para ir cerrando la brecha existente entre el desarrollo de la investigación científica, tecnológica y humanística existente, en comparación con los sistemas universitarios de otros países de la región. Empero, lo normativo no asegura una mejora significativa en la cantidad y la calidad de producción intelectual; depende, también, de la convergencia de otros factores o variables que se han identificado anteriormente; en especial, el desarrollo de toda una cultura investigativa mediante la implementación y ejecución de un conjunto de estrategias vinculadas con la docencia y el proceso enseñanza-aprendizaje en todos sus niveles (pregrado y posgrado) y en su modelo y dimensiones curriculares, como veremos seguidamente.

\section{Docencia de la investigación}

Uno de los problemas que enfrentan muchas universidades a nivel latinoamericano y en nuestro país, es no haber articulado políticas tendientes al fortalecimiento de la vinculación docencia e investigación. Situación que está siendo remontada, en la última década, en forma progresiva, por algunas universidades de países como Brasil, México y Colombia, entre otros; rompiendo muchos mitos sobre la actividad investigativa sostenida por siglos en la comunidad académica con expresiones como: "para hacer investigación se requiere personas con talentos especiales", "es muy difícil", "se requiere mucho tiempo", "se debe exigir en el posgrado", y en el síndrome "todo menos tesis" (TMT), imperante en los estudiantes de pregrado.

Coincidimos con otros autores en que la vinculación entre la docencia y la investigación debe empezar desde el pregrado y no 
esperar hasta el posgrado; debe tenerse en cuenta que investigar en la universidad connota dos aspectos. El primero, está asociado a los procesos de formación académico-profesional y, el segundo, con el desarrollo de una cultura de investigación, que entre otros elementos está constituida por los siguientes: un ambiente o clima institucional propicio para el desarrollo de la investigación en todos sus niveles y modalidades, la consolidación de comunidades académicas y científicas y la existencia de una área curricular sobre investigación, que posibilite el desarrollo de actitudes y aptitudes positivas hacia la construcción de conocimientos científicos de naturaleza disciplinar, interdisciplinar y transdisciplinar que permitan describir, explicar, predecir y/o controlar los fenómenos o aspectos de la realidad (natural, social, psicológica, educativa o virtual). Los profesores Mallart Navarra y Mallart Solaz (2017) de la Universidad de Barcelona lo expresan de este modo: "El profesorado que innova es un profesorado que investiga en su práctica. Hay mucho en común en ambas actuaciones" (p. 367).

En cuanto a la formación académico-profesional, la investigación científica va a contribuir a la mejora del proceso enseñanza aprendizaje moldeando el desarrollo de las cinco mentes necesarias para el presente milenio, propuestas por Gardner (2011), a saber: mente creadora, mente disciplinada, mente respetuosa, mente sintética y mente ética; así como las competencias específicas para la investigación: elaborar proyectos, plantear problemas a través de interrogantes, idear hipótesis, recopilar información, procesar datos, discutir, argumentar, interpretar, inferir y defender resultados, criticar la realidad, establecer contrastes y desarrollar autonomía. Lo antes señalado, es complementado por el mismo autor en un trabajo con colaboradores muy jóvenes, en el cual sostiene "que las tecnologías digitales han reconfigurado significativamente la identidad, la intimidad y la imaginación durante las últimas décadas" (2014, p.17). Con lo que se afirmaría que la educación no es posible en la actualidad fuera de la tecnología digital.

De otro lado, investigar para aprender, en el sentido amplio del constructo, implica preparar al potencial profesional para el control y el manejo de 
situaciones futuras, las cuales normalmente casi nunca tienen que ver con lo que se le enseña en un mundo cambiante e incierto como el que estamos viviendo, a fin de que los estudiantes no se queden con conocimientos cristalizados o esquematizados. Por el contrario, se apliquen estrategias sostenibles de intervención en su campo profesional.

En este sentido, el aprendizaje debe considerarse como una competencia indispensable que permita leer la realidad de diversas maneras, para así, enfrentarla más eficazmente en todos sus planos de interacción con creatividad; además, buscando el bienestar individual y colectivo.

De acuerdo a esto, el aprendizaje debe considerarse como una competencia indispensable que permita "leer" la realidad de diversas maneras y enfrentarla más eficazmente en todos sus planos de interacción con creatividad, en busca del bienestar individual y colectivo. Todo ello conlleva que el estudiante comprenda debidamente la espiral del aprendizaje, constituida por aprender, aprender a aprender, aprender a reaprender y aprender a desaprender, orientada al desarrollo de una actitud transformadora e innovadora.

Asimismo, resulta imperativo, destacar la relación existente entre investigación desarrollo e innovación $(\mathrm{I}+\mathrm{D}+\mathrm{i})$, que permite a las empresas y a los países incrementar el rigor en la aplicación de la ciencia y la tecnología y, por tanto, ser más competitivos; puesto que, les permitirá alcanzar mayores estándares de calidad y productividad. De allí, la necesidad de potenciar el papel de la investigación y la innovación aumentando el recurso humano dedicado a estas áreas, reforzando los derechos laborales de los investigadores.

En este sentido, más recientemente, en la conferencia mundial sobre ciencia para el siglo XXI auspiciada por la UNESCO y el Consejo Internacional para la Ciencia, se declara lo siguiente:

Para un país que quiere estar en condiciones de atender las necesidades fundamentales de su población, la enseñanza de la ciencia y la tecnología es un imperativo estratégico. Como parte de esa educación, los estudiantes deberán aprender a resolver problemas concretos y a atender las necesidades de la sociedad utilizando sus competencias y conocimientos científicos y tecnológicos (Programa en Pro de la Ciencia: Marco General de Acción, 1999). 


\section{Algunas estrategias para desarrollar una cultura investigativa}

A continuación, nos permitimos proponer algunas estrategias para desarrollar una cultura investigativa en la universidad. Entre las muchas existentes, proponemos las siguientes: la institucionalización de la investigación formativa, la alfabetización académica y los semilleros de investigación.

\section{a) La institucionalización de la investigación formativa}

La institucionalización de la investigación formativa se puede conseguir mediante políticas, lineamientos y normas claras, en especial de naturaleza curricular, que posibiliten el ligamen de la docencia con la investigación, facilitando la formación en y para la investigación, a través de actividades que no hacen parte necesariamente de un proyecto concreto. Su intención es la "formación" del estudiante en y para el proceso de investigación en todas sus fases: formal, lógica, metodológica y comunicativa.

La investigación formativa también puede denominarse, siguiendo a Parra (2004, p. 72): la enseñanza a través de la investigación o como docencia investigativa. Cada una de estas expresiones tiene un matiz propio: el primero, resalta la investigación como una técnica didáctica; el segundo, parece referirse más a una característica de la docencia y a un estilo docente. Sin embargo, las dos presentan un denominador común, que es su función o finalidad pedagógica. Agrega, asimismo, que la investigación formativa constituye una estrategia pedagógica de carácter docente para el desarrollo del currículo.

La función de este enfoque de investigación formativa es la de dar forma a la calidad, efectividad y pertinencia de la práctica o del programa, usualmente mientras una u otro están siendo llevadas a cabo (Restrepo, 2002); lo cual permite que tanto docentes (no acreditados como investigadores) y estudiantes, desarrollen habilidades cognoscitivas como el análisis, el pensamiento crítico, productivo y la solución de problemas que estas plantean; construyendo en los docentes la cultura de evaluación permanente de su práctica a través de procesos investigativos..

La institucionalización de la investigación ligada a la docencia, como un primer nivel de investigación en el pregrado, rompería con algunos mitos identificados anteriormente, que aún se vienen tejiendo en el contexto universitario. En este sentido, compartimos con Soria, (1985, p. 58) su 
propuesta de cinco supuestos sobre la docencia y la investigación:

1. La investigación, como toda actividad académica, es susceptible de enseñarse y de aprenderse en el salón de clase, en el laboratorio y en la comunidad.

2. La investigación, como proceso y como contenido, es el producto suma de conocimientos, valores, destrezas y habilidades, susceptibles de ser aislados, identificados y transmitidos de manera ordenada, secuencial y con un grado creciente de complejidad, de acuerdo con el nivel de desarrollo del estudiante.

3. La investigación puede ser introducida eficazmente en la docencia universitaria, clara y explícitamente formulada, como un constitutivo importante de la formación del joven estudiante.

4. La investigación puede nutrir los currículos, el contenido de las materias, las experiencias docentes, las actividades y las tareas diarias a lo largo de toda la carrera, desde el ingreso en el tercer nivel hasta la obtención del primer grado académico.

5. La identificación de disposiciones deseables para la investigación, susceptibles de ser enseñadas, pasa a ser tarea crucial para fomentar su estimulación pertinente.

\section{b) La alfabetización académica}

La alfabetización académica constituye un proceso de enseñanza de comunicación escrita de carácter académico de acuerdo a estándares disciplinarios; a través de la cual, los estudiantes aprenden a exponer, argumentar, resumir, buscar información, jerarquizarla, ponerla en relación, valorar razonamiento, debatir, etc. La alfabetización académica es una actividad transversal que incumbe a docentes, estudiantes e instituciones, desde todas las asignaturas, para favorecer el aprendizaje de las literalidades académicas (participación en todas las culturas escritas).

El concepto Alfabetización académica señala el conjunto de nociones y estrategias necesarias para participar en la cultura discursiva de las disciplinas, así como en las actividades de producción y análisis de textos, requeridas para aprender en la universidad (Carlino, 2005). Así mismo, desafía la creencia 
que el aprendizaje de la escritura consiste, únicamente, en la adquisición de una serie de habilidades que una vez desarrolladas pueden desplegarse en cualquier situación o contexto y postula que la lectura y la escritura son prácticas sociales que varían de acuerdo con las situaciones en las que se realizan (Lea y Creme, 2000).

Radloff y de la Harpe (2000) citados por Flórez y Gutiérrez (2011) definen la alfabetización académica como el proceso por el cual se llega a pertenecer a una comunidad científica y/o profesional, en virtud de haberse apropiado de sus formas de razonamiento instituidas a través de ciertas convenciones del discurso. Es una propuesta conceptual en la que se refieren las siguientes suposiciones sobre el proceso de escritura: a) la alfabetización es una habilidad de ingreso a la nueva cultura escrita; b) la escritura en la universidad es una adquisición espontánea frente al reconocimiento de que su aprendizaje requiere ser promovido por acciones institucionales; c) enseñar a escribir en la universidad es un proceso compensatorio/nivelatorio frente a la necesidad de hacer lugar en el currículo a las prácticas discursivas con las que cada disciplina elabora el conocimiento y d) la enseñanza de la escritura es asunto de especialistas frente a la necesidad de compromiso de toda la comunidad educativa (Carlino, 2005).

Asimismo, el concepto de alfabetización académica reconoce el valor epistémico de la escritura y su importancia en la consolidación de comunidades académicas y de discurso. De la misma forma, reconoce que escribir involucra una serie de actos esenciales que van desde las habilidades comunicativas básicas hasta la apropiación de contenidos disciplinares. En este sentido, es importante precisar que la alfabetización académica es un concepto que intenta armonizar posturas, algunas veces polarizadas, sobre lo que significa escribir en la universidad.

\section{c) Los semilleros de investigación}

Los semilleros de investigación son una estrategia de aprendizaje, basada en el principio de aprender haciendo, donde un conjunto de estudiantes interesados por un tema lo abordan haciendo uso del método científico y comunican sus hallazgos bajo formatos estandarizados. Institucionalmente, los semilleros de investigación operan en función a líneas de investigación específicas. 
Los semilleros de investigación son espacios que permiten ejercer la libertad y la crítica académica, la creatividad y capacidad de asombro, formar, capacitar y transformar masa crítica investigativa y promover escuelas de pensamiento.

Son también comunidades de aprendizaje de estudiantes y profesores, de una o de diferentes disciplinas, surgidas en el seno de la Universidad por el interés en investigación de los actores que los integran. Como comunidades de aprendizaje se configuran, en el plano de la conformación, por un sentido de pertenencia enmarcado en lo heterogéneo, lo diverso, lo abierto, lo dinámico y lo flexible; $y$, en el plano de la pervivencia, por un sentido de pertinencia enmarcado por lo planeado, lo estratégico, lo guiado, y lo que en últimas determina para el semillero de investigación un ser y estar en el mundo; es por esto que tienen unos objetivos y un plan de acción a mediano o largo plazo, que responden a las necesidades del semillero y de la Institución.

Los semilleros se articulan como comunidad académica al sistema de investigación de la Universidad, a través de su vinculación con los grupos de investigación y los centros de excelencia, lo que los convierte en las células de formación del relevo generacional de los investigadores. De igual manera, se vinculan con los pregrados y posgrados de la universidad, ya que, sus áreas de trabajo se derivan de los intereses que surgen en los estudiantes en el desarrollo formal de sus estudios. Los semilleros de investigación pueden estar conformados, en principio, por profesores - investigadores, estudiantes de posgrado y pregrado, o por profesionales. Los miembros pueden ser tanto personas de la universidad como personas externas a ella, siempre y cuando estén vinculadas al semillero a partir de un acuerdo interinstitucional.

El programa de semilleros de investigación se establece como factor aglutinador de los diferentes semilleros que existen en la institución y sigue el esquema de trabajo de una red académica. Entre los objetivos principales del programa están los siguientes:

- Fortalecer la estructura del sistema de investigación en la universidad a través del fomento a la creación de semilleros y consolidación de los mismos.

- Fomentar la investigación formativa y la investigación propiamente dicha entre los estudiantes. 
- Estimular la pregunta en la cotidianidad universitaria a través de espacios dentro y fuera del aula de clase, que contribuya al desarrollo de una cultura de aprendizaje.

- Fortalecer la relación academia-investigación, propiciando la interacción multidisciplinar de estudiantes, profesores e investigadores.

- Generar cultura investigativa en la universidad a través de la creación de espacios que acerquen a los estudiantes a la investigación y le muestren esta como una de sus opciones profesionales.

- Generar mecanismos que permitan la vinculación de estudiantes con los grupos de investigación como parte del proceso de relevo generacional de profesores e investigadores.

- Conformar y facilitar el surgimiento de una red de semilleros de investigación en la universidad, que a su vez participe en redes nacionales e internacionales.

\section{Conclusiones}

- La formación académico-profesional y el desarrollo de una cultura de investigación, son factores condicionantes para el desarrollo de habilidades investigativas que permitan describir, explicar, predecir y/o controlar los fenómenos o aspectos de la realidad.

- La alfabetización académica, al ser un proceso de enseñanza de comunicación escrita de carácter académico de acuerdo a estándares disciplinarios, a través de la cual los estudiantes aprenden a exponer, argumentar, resumir, buscar información, jerarquizarla, ponerla en relación, valorar razonamiento, debatir, etc. debería ser una actividad transversal que incumbe a docentes, estudiantes e instituciones, desde todas las asignaturas, para favorecer el aprendizaje de las literalidades académicas (participación en todas las culturas escritas).

- Los semilleros se articulan como comunidad académica al sistema de investigación de la universidad, a través de su vinculación con los grupos de investigación y los centros de excelencia, lo que los convierte en las células de formación del relevo generacional de los investigadores. 


\section{Referencias}

Carlino, P. (2005). Escribir, leer y aprender en la universidad. Una introducción a la alfabetización académica. Buenos Aires: Fondo de Cultura Económica.

Flórez, R. y Gutiérrez, M (2011). Bogotá: Universidad Nacional de Colombia

Gardner, H (2011). Las 5 mentes del futuro. Barcelona: Paidós Ibérica.

Gardner, H. y Davis, K. (2014). La generación APP. Cómo los jóvenes gestionan su identidad, su privacidad y su imaginación en el mundo digital. Barcelona: Espasa Libros.

Lea, M y Creme, P. (2000). Escribir en la universidad. Barcelona: Gedisa.

Mallart, J. y Mallart, A. (2017). Formación del profesorado para la investigación y la innovación en ecoformación. Barcelona: Gedisa.

Medina, A., De la Herrán, A. y Domínguez, M. C. (Coord.). Nuevas perspectivas en la formación de profesores. (pp. 363-420). Madrid: UNED.

Ministerio de Educación. (2006). La universidad en el Perú. Razones para una Reforma Universitaria. Lima: MED.

Ministerio de Educación. (2014). Ley Universitaria $N^{\circ}$ 30220. Lima. Disponible http://repositorio.minedu.gob.pe/handle/123456789/321

Parra, C. (2004). Apuntes sobre la investigación formativa. Educación y Educadores, 7, 57 -77.

Navarra, M. y Solaz, M. (2017). De la educación para el desarrollo sostenible a la Ecopedagogía. Barcelona: Hergué.

Quintanilla, M (2007). La investigación en la sociedad del conocimiento. Rev. iberoam. cienc. tecnol. soc. v.3 n.8 Ciudad Autónoma de Buenos Aires abr. 2007. 
Restrepo, B. (2002). Conceptos y aplicaciones de la investigación formativa y criterios para evaluar la investigación en sentido estricto. Bogotá: CNA.

Soria, O. (1985). Docencia de la investigación en la Universidad. Revista Interamericana de Desarrollo Educativo, No 98, Año XXX. México.

Tünnermann, C, y de Souza Chaui, M. (2003). Desafíos de la universidad en la Sociedad del conocimiento, cinco años después de la Conferencia Mundial sobre Educación Superior. Disponible. nesdoc.unesco.org/.../ ulis.pl?...Tünnermann\%20Bernheim...

UNESCO (2005). Hacia las sociedades del conocimiento. París: UNESCO. Disponible.unesdoc.unesco.org/images/0014/001419/141908s.pdf

UNESCO-IESALC (2018). Declaración de la CRES 2008. Disponible www. cres2018.org/historia/cres-2008. 